\title{
Reabilitação funcional e estética de paciente com dentição desgastada: uma abordagem minimamente invasiva
}

\author{
Functional and aesthetic rehabilitation of patient with worn \\ dentition: a minimally invasive approach
}

\section{RESUMO}

Introdução: O desgaste generalizado dos dentes pode levar à deterioração do plano oclusal levando a um comprometimento estético-funcional dos dentes e a quadros complexos de reabilitação oral. Caso Clínico: Paciente SSS, homem, 62 anos, saúde sistêmica estável, dentado total, com sinais clínicos de desgaste dental generalizado por atrição. Foi confeccionada uma placa (overlay) estabilizadora para aumentar a DVO do paciente, gerando o espaço necessário para a reconstrução das estruturas perdidas. Posteriormente foram restaurados com resina composta direta todos os elementos do arco inferior, bem como os elementos 11; 12; 13; 14; 21; 22; 23 e 24 no arco superior. Desta forma se reestabeleceu, forma, função e estética dos elementos restaurados com uma abordagem conservadora e de baixo custo. Um dispositivo interoclusal foi confeccionado a fim de evitar a degradação dos dentes restaurados. Conclusão: Esse caso demonstrou um tratamento restaurador minimamente invasivo com a utilização de restaurações diretas em resina composta, o qual pode ser considerado como uma alternativa para a reconstrução de dentição desgastada por atrição, apresentando excelente resultado em curto prazo.

Palavras-chave: Desgaste dental, Reabilitação Oral, Restauração Direta.

\begin{abstract}
Introduction: Generalized worn dentition may lead to occlusal plane deterioration, causing compromising of esthetics and function of the dentition, what con lead to complex oral rehabilitation scenarios. Clinical study: Patient SSS, 62 years old, stable systemic health, with all teeth, presenting generalized tooth wear caused by attrition. As part of the treatment, a removable overlay has been used to increase the patient's OVD and allowing for the needed space for tooth reconstruction. afterwards all elements of the lower dental arch as well of tooth \#11;12;13;14;21;22; 23 and 24 , were restored with the use of direct composite resin. With this all damages affecting the form, function and aesthetics of the involve elements have been corrected with a minimally invasive and low cost approach. Conclusion: This case demonstrated a minimally invasive restorative treatment with the use of direct restorations in composite resin, which can be considered as an alternative for reconstruction of attrition-worn dentition, presenting excellent short-term results.
\end{abstract}

Key words: Dental Wear, Oral Rehabilitation, Direct Restoration

\section{Gisele Heise $^{1}$ \\ Juliana Keiko Prado Kiguti ${ }^{1}$ \\ Brunna Katyuscia de Almeida \\ Guanaes $^{2}$ \\ Fernando Ruppel Osternack ${ }^{3}$ \\ Gisele Maria Correr Nolasco ${ }^{3}$ \\ Carlos Eduardo E. Rezende ${ }^{3}$}
${ }^{1}$ Aluno de Graduação em Odontologia, Univer- sidade Positivo
${ }^{2}$ DDS, Mestranda em Odontologia Clinica,
Universidade Positivo
${ }^{3}$ DDS, MSc, PhD, Professor do curso de Graduação em Odontologia, Universidade Positivo

Autor correspondente:

Carlos Eduardo E. Rezende

Rua: Prof. Pedro Viriato Parigot de Souza, 5300

- Cep 81280-330

Curitiba/ PR

Telefone: 3317-3000

Email: caerezende@gmail.com 


\section{INTRODUÇÃO}

Frequentemente os cirurgiões-dentistas se deparam com casos clínicos em que há o desgaste dos tecidos dentários ${ }^{1}$. A dentição desgastada leva a problemas clínicos como sensibilidade dentária, comprometimento estético, redução da capacidade mastigatória, perda de espaço inter-oclusal e perda das guias anteriores (oque pode levar a prejuízos ao periodonto de sustentação dos dentes posteriores e acelerar a perda de tecido dentário) ${ }^{2}$. Tal desgaste pode ocorrer a partir de lesões cariosas ou não cariosas. As primeiras podem ocorrer pela ação de ácidos oriundos de bactérias causadoras da cárie. Já as lesões não cariosas apresentam quatro processos etiológicos principais, sendo eles: abrasão, erosão, abfração e atrição. ${ }^{3}$

A atrição se caracteriza pelo desgaste das superfícies dos dentes pelo contato direto com os seus dentes antagonistas ${ }^{3}$. Esta pode ocorrer durante movimentos de mastigação, tendo como resultado leve perda da estrutura dental, sendo assim considerada como fisiológica. Porém, também pode ser consequência de um ranger exacerbado dos dentes e então se torna uma patologia, cuja severidade tem correlação direta com hábitos parafuncionais denominados apertamento ou bruxismo associados à força aplicada, frequência e tempo ${ }^{4}$.

As lesões por atrição acometem as superfícies de contato entre os dentes superiores e inferiores, assim sendo, as faces afetadas durante este processo são oclusais, incisais e a palatina de dentes anterossuperiores. ${ }^{3} \mathrm{~A}$ atrição desencadeia o desgaste em facetas coincidentes nos dentes antagonistas. ${ }^{5} \mathrm{O}$ desgaste dos dentes é, de forma geral, uniforme, porém existem casos em que podemos identificar a discrepância de resistência ao desgaste em áreas de um mesmo elemento, denotando a variação da composição interna do dente. Ou seja, as áreas de maior mineralização resistem mais ao desgaste. ${ }^{3,4}$

O desgaste provocado pela atrição nos elementos posteriores pode, em certos casos, levar à redução da dimensão vertical de oclusão (DVO). Em geral, a redução da DVO não é percebida pelos pacientes, mas quando os dentes anteriores são atingidos e ocorre a exposição da dentina mais escura que o esmalte, os pacientes buscam pelo cirurgião dentista ${ }^{6}$. A redução da DVO ocorre quando o desgaste dos dentes ocorre mais rapidamente do que a extrusão compensatória dos dentes, a qual seria capaz de manter a DVO ${ }^{6}$. Mesmo quando há a extrusão compensatória e manutenção da DVO, a reconstrução da estrutura dentária perdida por motivos estéticos pode ser um desafio clínico, uma vez que não haveria espaço interoclusal disponível para tal reconstruçãoํ.

Para circunstâncias em que o paciente necessita de restabelecimento da dimensão vertical de oclusão, bem como da recuperação de forma e função dos elementos, existem algumas alternativas recomendadas para a reabilitação oral do paciente, entre elas a prótese parcial removível tipo overlay. ${ }^{7,8}$ Outra alternativa terapêutica seria a utilização de próteses fixas (coroas indiretas), a qual requer maior desgaste do elemento dentário, maior tempo de tratamento e maior custo agregado, porém com ótimos resultados estéticos. ${ }^{9}$.

Uma alternativa mais acessível do ponto de vista financeiro e menos invasiva do ponto de vista biológico (não necessita de desgaste acentuado das estruturas dentárias) seria a reconstrução direta em resinas compostas ${ }^{10}$.

O objetivo deste trabalho é relatar um caso clínico de desgaste generalizado dos dentes por atrição patológica que desencadeou a perda da forma e função dos dentes 
anteriores e posteriores dos dois arcos dentais, além da restrição do espaço interoclusal disponível para a reconstrução estética dos tecidos perdidos, a partir de abordagem restauradora conservadora e de baixo custo.

\section{RELATO DE CASO}

Paciente S.S.S., 62 anos, residente na cidade de Curitiba-PR, gênero masculino, profissional da construção civil, procurou a Clínica Odontológica da Universidade Positivo com a queixa principal de melhorar a estética dos dentes, pois eles estavam "muito desgastados e escuros". Durante a consulta inicial e anamnese o paciente relatou saúde sistêmica estável e a não utilização de terapia medicamentosa. Na avaliação clínica inicial observou-se a presença dos terceiros molares 18 e 28 e a ausência dos dentes 38 e 48, o paciente apresentou saúde gengival satisfatória, salivação dentro de padrões de normalidade, padrão oclusal de mordida profunda e redução do espaço interoclusal, o desgaste das superfícies oclusal, incisal dos dentes inferiores e superiores, além de desgaste palatino dos dentes ântero-superiores (Figura 1). Foi constatada a contribuição a perda de estrutura mineral por atrição, decorrente do hábito parafuncional (bruxismo) sem a utilização de dispositivos (placas) oclusais, sendo essa a causa principal do desgaste moderado dos dentes.

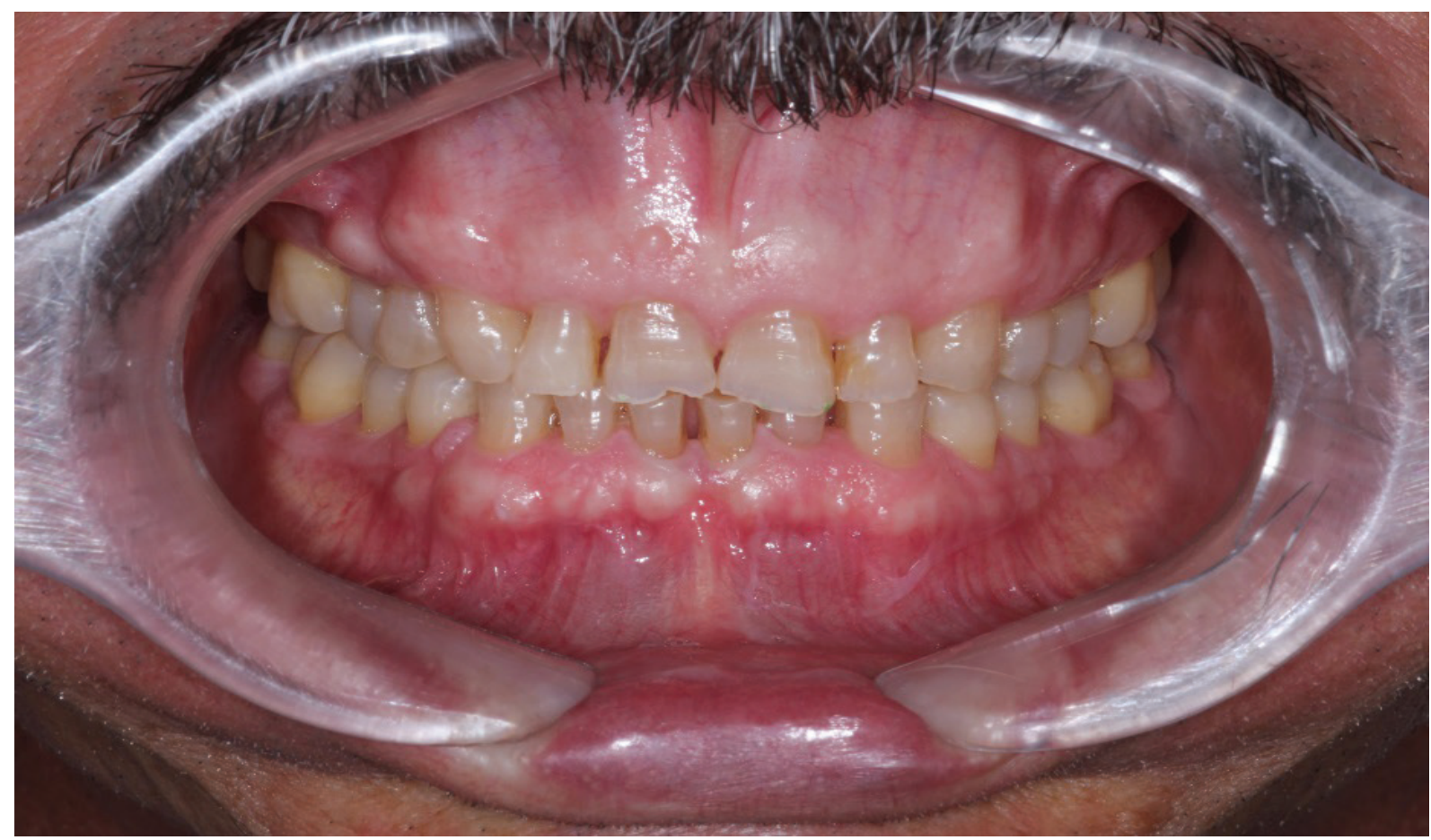

Figura 1 - Fotografia inicial, observa-se o desgaste das inicisais dos dentes anterossuperiores, bem como a mordida profunda. 


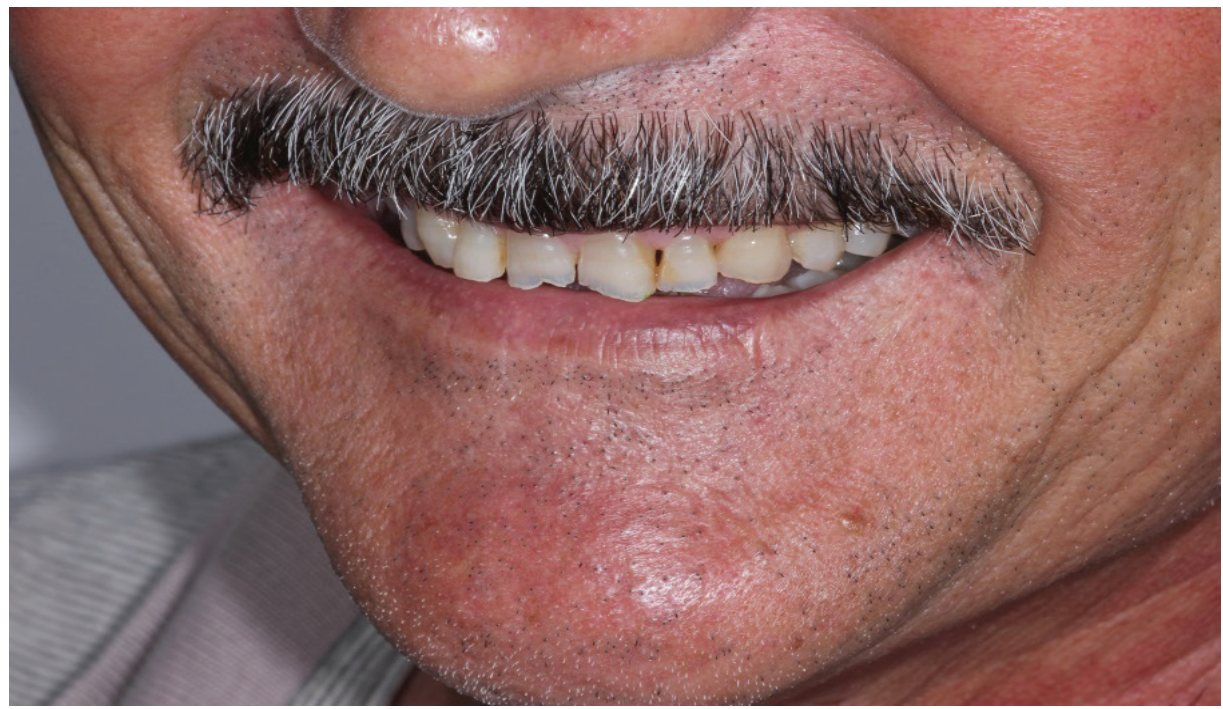

Figura 2 - Fotografia inicial vista lateral, observa-se o desgaste das inicisais dos dentes anterossuperioes

O exame radiográfico panorâmico (Figura 3) foi solicitado para diagnóstico complementar. A partir dele foi possível avaliar a favorável inserção óssea dos dentes, sem espessamento dos ligamentos periodontais, as restaurações dos elementos 35, 36 e 46, quais se apresentavam bem adaptadas e o posicionamento de extrusão dos terceiros molares 18 e 28.

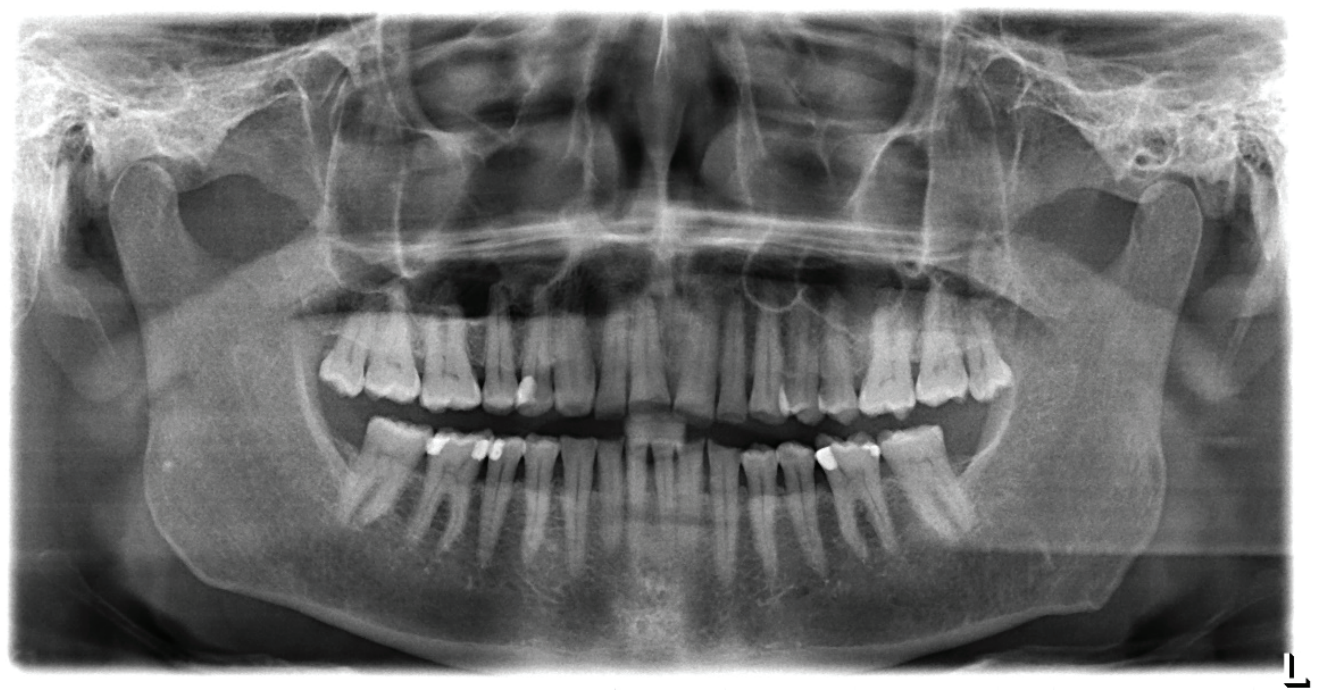

Figura 3 - Radiografia Panorâmica, fonte para exame complementar.

\section{PROCEDIMENTOS REABILITADORES - SEQUENCIA CLÍNICA}

O inicio do tratamento se deu a partir da moldagem de estudo em alginato (Cavex Colorchange, Fustweg - Holanda) para a análise dos modelos em articulador semi-ajustável (ASA). Durante a manipulação do paciente foi constatado que os terceiros molares 
apresentavam contato prematuro durante a oclusão, assim os elementos 18 e 28 foram extraídos, uma vez que não possuíam dentição antagonista.

Constatada a redução do espaço interoclusal buscou-se recuperar este espaço, pois sem esse levantamento da oclusão não seria possível iniciar o processo restaurador. Assim, se estabeleceu a dimensão vertical de oclusão (DVO), a partir da dimensão vertical de repouso (DVR) pelo uso do compasso de Willis. Em seguida, foi confeccionado um Jig para auxiliar o registro intermaxilar na nova DVO na posição de relação cêntrica (RC). A nova DVO foi conferida pela relação das proporções faciais e testes fonéticos.

O arco facial foi montado e os modelos foram montados em ASA na nova DVO em posição de RC. A montagem dos modelos foi conferida para se certificar que estava semelhante à posição de RC obtida clinicamente. Tal montagem foi enviada ao laboratório para confecção de uma placa para restabelecimento de dimensão vertical (Figura 4 A e B), para averiguação estética e funcional da nova condição dos dentes. A placa foi instalada provisoriamente (Figura 5) e o paciente foi instruído a utilizá-la durante todo o dia, por trinta dias, retirando-a apenas para dormir. Durante este período, houve retornos periódicos para ajustes oclusais.
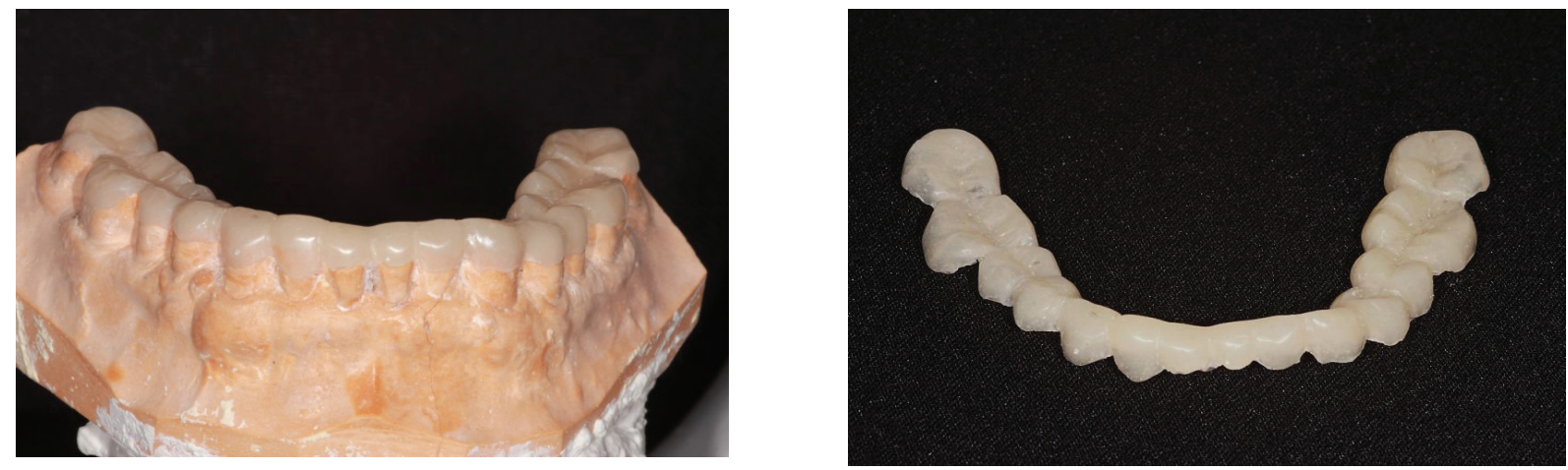

Figura 4 A e B Placa de estabilização (overlay) para que o paciente se habitue à nova DVO.

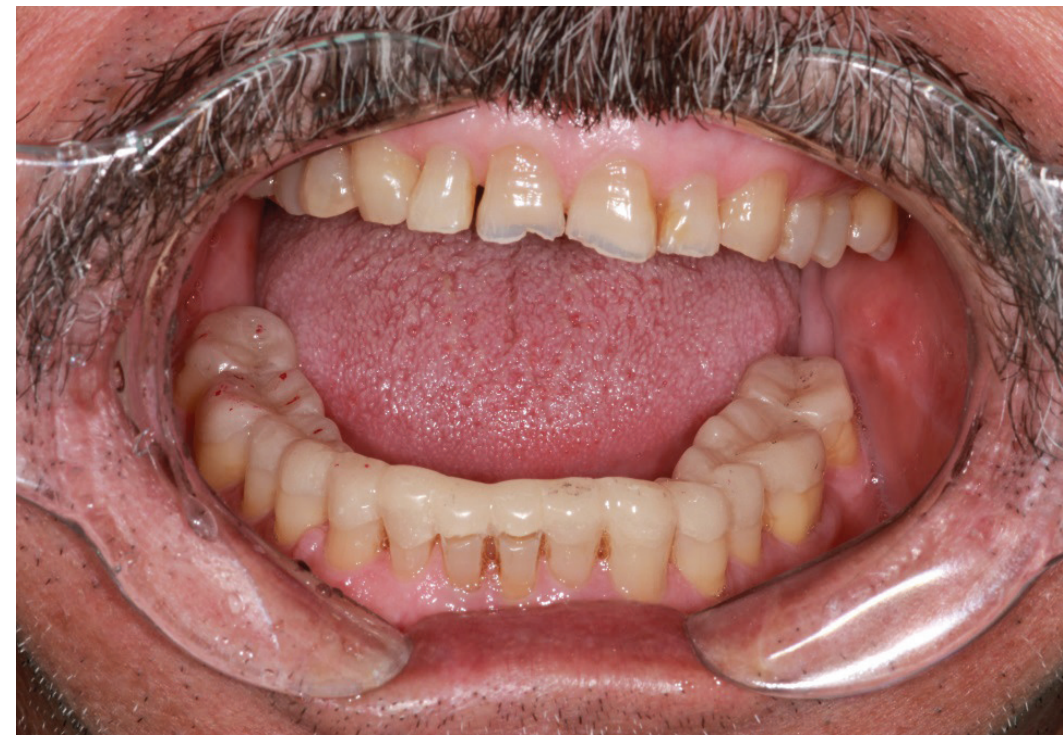

Figura 5 - Placa de estabilização, instalação e ajustes dos pontos de contato. 
Passados trinta dias de utilização do dispositivo interoclusal efetuou-se a restauração das faces oclusais de todos os elementos do arco inferior com resina composta. Para tal feito foi fabricado um mock-up de acetato de $1 \mathrm{~mm}$ para que a luz ultrapassasse o material e fosse possível polimerizar a resina depositada na coroa dental. Os elementos foram condicionados com ácido fosfórico a 37\%, posteriormente a lavagem e secagem aplicou-se sistema adesivo Adapter Single Bond 2 - 3M. O processo restaurador se deu através de resina composta fotopolimerizável na cor A2 da marca comercial (Charisma Diamond - Kulzer). Após o arco inferior ter sido restaurado, uma nova moldagem foi efetuada e enviada ao laboratório para então se efetuar o enceramento diagnóstico dos elementos anteriores superiores e inferiores (Figura 6).

O enceramento diagnóstico possibilitou a visualização dos resultados estéticos a partir de um mock-up com a utilização de resina bisacrílica (Structur - VOCO). Após a aprovação do paciente quanto à estética dos dentes anteriores, procedeu-se a reconstrução dos mesmos a partir do uso de uma matriz feita de silicone transparente (Zhermack - Elite Transparent). Assim, os elementos anterossuperiores (13 ao 23) e os anteroinferiores (33 ao 43) foram restaurados. A superfície dos dentes foi condicionada e adesivada da forma já descrita anteriormente e então o processo restaurador aconteceu de forma isolada por elemento.

Os compósitos de resina foram inseridos no mock-up o mesmo foi inserido na cavidade bucal e posicionado sobre o dente para, em seguida, executar a fotopolimerização por 20 segundos em cada face do dente envolvida. Após retirar o guia de silicone a resina estava devidamente assentada e aderida ao esmalte. Os ajustes foram efetuados com brocas de ponta diamantada cônica de extremidade arredondada (FG 3195FF - KG Sorensen) para as faces proximais e brocas diamantada de ponta de chama (3118 FF - KG Sorensen) para as regiões lingual e palatina. Tiras de lixa para polimento auxiliaram na delimitação dos pontos de contato. A oclusão foi checada com o auxílio do papel carbono e os ajustes necessários foram realizados. Os elementos foram polidos com borrachas abrasivas, pasta para polimento e escova de pelos de cabra. (Figura 7 A e B)

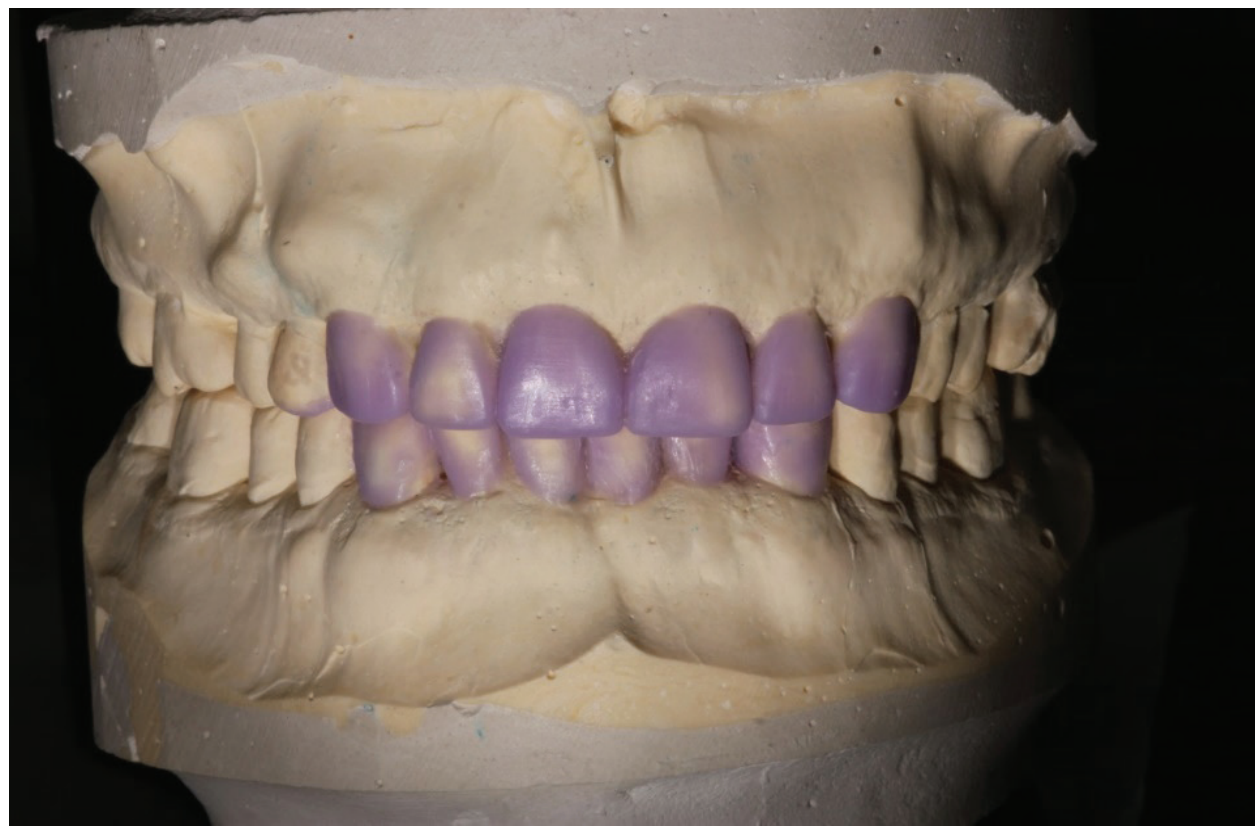

Figura 6 - Enceramento diagnóstico para reconstrução estética do novo sorriso. 

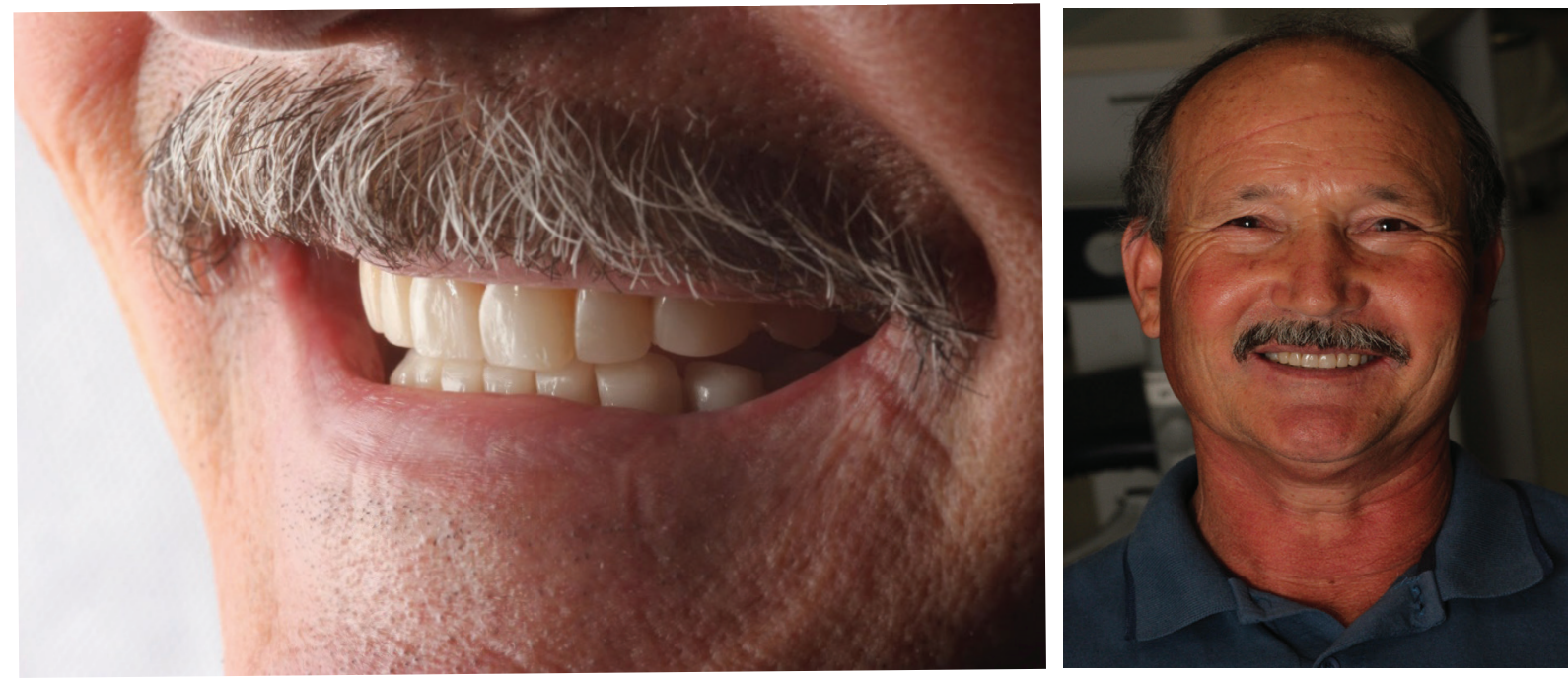

Figura 7 A e B - Aspecto final do tratamento reabilitador, vista intra-oral (A) e extra-oral (B).

O tratamento foi finalizado com a confecção de um dispositivo interoclusal para que o paciente utilize todas as noites durante o sono, de forma a garantir maior longevidade das restaurações.

\section{DISCUSS ÃO}

Este trabalho relatou o caso clínico de um paciente com desgaste extenso das faces oclusais e incisais dos dentes do arco superior e inferior. Estes desgastes causavam descontentamento estético ao paciente. A abordagem realizada baseou-se num processo minimamente invasivo e na estratégia mais conservadora possível para reestabelecer o espaço interoclusal perdido e a estética desejada, aliados ao menor custo financeiro possível para o paciente. Apoiar o tratamento a técnicas que preconizem a restauração dos dentes utilizando pouco desgaste dental e baixo custo possibilitam ao paciente baixo custo financeiro e biológico. Assim, optou-se por instalar uma placa interoclusal estabilizadora provisória com a função de reequilibrar o posicionamento mandibular, estabilizando a oclusão ${ }^{12}$ e fazendo com que o paciente se adaptasse à nova dimensão vertical de oclusão. Posteriormente os elementos foram restaurados com resina composta para que se obtivesse o restabelecimento do formato da anatomia dental e melhoria da função e estética.

A evolução dos materiais restauradores e dos sistemas adesivos odontológicos melhorou o desempenho clínico das restaurações estéticas posteriores, assim como possibilitou novas estratégias para dentes anteriores, aliados ao menor desgaste dos dentes ${ }^{13}$. $\mathrm{O}$ planejamento foi baseado em evidências e nas propriedades dos materiais disponíveis para que o material fosse selecionado de forma a agregar um bom custo benefício ao tratamento proposto. ${ }^{11,13}$. As reconstruções com resinas compostas para a reabilitação de pacientes com dentição desgastada têm sido discutidas na literatura ${ }^{11,13,14}$. O sucesso em longo prazo deste tipo de reabilitação foi demonstrado no estudo de Gulamali et al. $2011{ }^{15}$, comprovando a reconstrução com resinas compostas como uma alternativa confiável para tais casos. As complicações decorrentes de tal tipo de reabilitação são, geralmente, passíveis de reparo, 
sem que haja a substituição precoce das restaurações. As complicações mais frequentemente relatadas são o lascamento da resina, principalmente em áreas onde há pouco, ou nenhum esmalte na margem da restauração ${ }^{16,17}$. Meyers $2013^{14}$ destacou a importância da manutenção pós-tratamento para o sucesso em longo prazo das reconstruções de dentes desgastados em resina composta, a fim de reduzir a possibilidade de complicações que indicassem a substituição das restaurações.

Outros conceitos foram levados em consideração, um deles foi o Conceito de Dahl. Em 1975, Dahl ${ }^{18}$ relatou o uso de um dispositivo oclusal anterior removível de cobalto-cromo para gerar espaço interoclusal para posterior restauração de dentição desgastada. O autor observou uma combinação de intrusão ortodôntica dos dentes anteriores e erupção dos dentes posteriores, reduzindo a necessidade de restaurações nos dentes e, concluiu, relatando que as relações verticais eram praticamente estáveis ${ }^{18}$. Hoje em dia, esta técnica é substituída pelo uso da resina composta ao invés de um dispositivo de cobalto-cromo ${ }^{15}$. Este conceito não se enquadrou para o tratamento do caso citado devido ao longo período que o paciente teria que dispor com o dispositivo para que ocorresse a extrusão desejada, pois o paciente iria mudar de cidade dentro de 60 dias e, portanto, gostaria de ter seu caso solucionado dentro deste prazo.

Outra opção para o tratamento reabilitador seriam as próteses fixas em metalocerâmica, mas para que uma prótese preencha os pré-requisitos estéticos e funcionais, é necessário que o desgaste dental proporcione espaço para o metal, opaco e cerâmica ${ }^{14,19}$, além de apresentar um custo mais elevado. Desta forma, o paciente não dispunha de verba e não desejava o desgaste dos dentes para o preparo das peças protéticas, inviabilizando esta opção de tratamento.

A PPR também seria uma opção econômica ${ }^{7-8}$, porém tal opção foi descartada, pois o paciente não aceitou os valores propostos e não se sentia seguro em usar um dispositivo removível. Assim, a melhor solução considerada para o caso, foi a que apresentaria um menor tempo de preparo e menor custo, com a confecção de uma PPR overlay provisória para recuperação do espaço oclusal e posterior restauração das estruturas desgastadas em resina composta direta.

A etiologia do desgaste dentário também deve ser avaliada, de forma a evitar possíveis fracassos precoces do trabalho restaurador executado. Neste caso em específico, foi constatada atrição por bruxismo noturno, assim, foi indicada a utilização de dispositivo interoclusal em resina acrílica de forma a se evitar o desgaste das restaurações de resina. É importante salientar que o planejamento do caso deve levar em consideração as particularidades de cada caso, seguindo as expectativas dos pacientes ${ }^{20}$. Além disso, o conhecimento dos princípios básicos de oclusão e prótese são fundamentais para que o profissional possa planejar e conduzir as etapas clínicas da maneira mais adequada.

\section{CONCLUSÃO}

A abordagem clínica oferecida ao paciente proporcionou restabelecimento de padrões aceitáveis de função, estética, conforto e saúde, cumprindo com a necessidade inquirida pelo paciente. Assim, concluímos que os tratamentos restauradores minimamente invasivos, com pouco ou zero desgaste dos dentes, baseados na adesão aos tecidos dentários têm excelente resultado funcional e estético a curto prazo. 


\section{REFERÊNCIAS}

1. DAVIES SJ, GRAY RJM, Qualtrough AJE. Management of tooth surface loss. Br Dent J 2002;192:11-23.

2. MUTS EJ, VAN PELT H, EDELHOFF D, KREJCI I, Cune M. Tooth wear: a systematic review of treatment options. J Prosthet Dent. 2014;112(4):752-9. doi: 10.1016/j.prosdent.2014.01.018

3. FILHO WG.; SILVA VA. Lesões Não Cariosas: “O novo desafio da Odontologia". 1ª ed. São Paulo: Editora Santos 2008.

4. $\quad$ VERRETT RG. Analyzing the Etiology of an Extremely Worn Dentition. J Prosthodont. 2001;10(4):224-33.

5. MOSHAVERINIA A, KAR K, AALAM AA, TAKANASHI K, KIM JW, CHEE WW. A multidisciplinary approach for the rehabilitation of a patient with an excessively worn dentition: A clinical report. J Prosthet Dent. 2014;111(4):259-63. doi: 10.1016/j.prosdent.2013.11.006.

6. DANTAS EM. A importância do restabelecimento da dimensão vertical de oclusão na reabilitação protética; Odonto 2012; 20(40): 41-48.

7. FREITAS R.; KAISER O.; B. PIGOSO MN.; CAVALLARI P.; RESENDE DRB. Diagnóstico e Tratamento da Dimensão Vertical de Oclusão Diminuída. RGO 2006; 54(2):161-164.

8. SATO S.; Hotta TH.; Pedrazzi V. Removable occlusal overlay splint in the management of tooth wear: a clinical report. J Prosthet Dent. 2000, 83(4):392-395.

9. MOSLEHIFARD E, NIKZAD S, GERAMINPANAH F, MAHBOUB F. Full-mouth rehabilitation of a patient with severely worn dentition and uneven occlusal plane: a clinical report. J Prosthodont. 2012; 21(1):56-64. doi: 10.1111/j.1532-849X.2011.00765.x.

10. PINHEIRO SL, LOPES MF, PINHEIRO MH, CARVALHO WL, PINHEIRO IVAP. Reabilitação estética simplificada em dentes anteriores desgastados pelo bruxismo: relato de caso. Full Dent. Sci.2011; 2(8):421-427.

11. POYSER NJ.; BRIGGS FA BRIGGS.; CHANA HS.; KELLERHER MGD.; PORTER RWJ.; PATEL M. The evaluation of direct composite restorations for the worn mandibular anterior dentition - clinical performance and patient satisfaction. J Oral Rehabil. 2007;34(5):361-76

12. PAIVA HJ. Oclusão Noções e Conceitos Básicos; 1. ed. São Paulo: Editora Santos, 1997, p. 219-225.

13. BALDISSERA RA, CORREA MB, SCHUCH HS, Collares K, Nascimento GG, Jardim PS, Moraes RR, Opdam NJ, Demarco FF. Are there universal restorative composites for anterior and posterior teeth? J Dent. 2013;41(11):1027-35.

14. MEYERS IA. Minimum intervention dentistry and the management of tooth wear in general practice. Aust Dent J. 2013;58 Suppl 1:60-5. doi: 10.1111/adj.12050.

15. GULAMALI AB. HEMMINGNS KW, TREDWIN CJ, PETRIE A. Survival analysis of composite Dahl restorations provided to manage localized anterior tooth wear (ten year follow-up). Br Dent J. 2011; 26;211(4):E9. doi: 10.1038/sj.bdj.2011.683.2

16. BURKE FJ, KELLEHER MG, WILSON N, BISHOP K. Introducing the concept of pragmatic esthetics, with special reference to the treatment of tooth wear. J Esthet Restor Dent. 2011; 23(5):277-93. doi: 10.1111/j.1708-8240.2011.00462.x.

17. HEMMINGS KW, DARBAR UR, VAUGHAN S. Tooth wear treated with direct composite restorations at an increased vertical dimension: results at 30 months. J Prosthet Dent. 2000; 83:287-293.

18. DAHL BL, KROGSTAD O, KARLSEN K. An alternative treatment in cases with advanced localized attrition. J Oral Rehabil. 1975; 2:209-214.

19. BONFANTE, G. ; BATISTA, J. G. ; PANTALEÓN, D. S. . Fatores estéticos no preparo do dente para coroas metalocerâmicas. Revista Brasileira Prótese Periodontia Oclusão 1995; 1(1/3): 1-19.

20. DA CUNHA MC, SANTOS JF, SANTOS MB, MARCHINI L. Patients' Expectation Before and Satisfaction After Full-Arch Fixed Implant-Prosthesis Rehabilitation. J Oral Implantol. 2015; 41(3):235-9. doi: 10.1563/ AAID-JOI-D-12-00134. 